\title{
Multiple nocardial brain abscesses in an immunocompromised patient with myasthenia gravis
}

\author{
Ayesha Shaikh, Maria Lola Cevallos, Fang Lan, Jean Pratt Daniel
}

\begin{abstract}
Introduction: Nocardia are gram positive, variably acid-fast positive diphtheroidlike to branched, filamentous, aerobic actinomycetes. Nocardiosis is an opportunistic infection that has been noted in patients with malignancies, systemic lupus erythematosus, HIV infection, hematopoietic stem cell transplant recipients and long-term steroid users. Case Report: A 32year-old female presented with history of myasthenia gravis on long-term glucocorticoid therapy. During her last admission, wound culture of her left shoulder abscess showed diptheroid organisms. Patient presented with severe headache, nausea, vomiting and altered mental status. She was initially diagnosed with metastatic cerebral abscess and treated with empiric antimicrobial therapy. Imaging study of the brain showed bilateral occipital ring enhancing lesions. Biopsy results came back as culture positive for nocardia. Patient was subsequently treated with intravenous antibiotics for a total of six months. Conclusion: Cases of nocardiosis may go undiagnosed, either because they respond to empiric antimicrobial
\end{abstract}

Ayesha Shaikh¹, Maria Lola Cevallos², Fang Lan ${ }^{3}$, Jean Pratt Daniel ${ }^{4}$

Affiliations: ${ }^{1}$ PGY1, Internal Medicine Lincoln Medical and Mental Health Center, Bronx, NY, United States; 2Department of Internal Medicine, Lincoln Medical \& Mental Health Center, Bronx, NY, United States; ${ }^{3}$ PGY2 , Internal Medicine, Lincoln Medical and Mental Health Center, Bronx, NY, United States; ${ }^{4}$ Department of Internal Medicine, Lincoln Medical \& Mental Health Center, Bronx, NY, United States.

Corresponding Author: Ayesha Shaikh, MD 234 East 149th Street, Bronx, New York, USA-10451; Ph: 1-718-5796429; Fax: 1-718-579-4836; Email : ashaikhdr@gmail.com

Received: 03 November 2011

Accepted: 27 March 2012

Published: 01 January 2013 treatment or because Nocardia spp. may be difficult to identify in cultures of clinical specimens. They may be mistaken for nonpathogenic microorganisms (diphtheroids) and discarded. High suspicion and early long term institution of therapy are key to a favorable outcome of this disease which has high mortality rates.

Keywords: Nocardiosis, Brain, Abscess, Steroid use

$$
* * * * * * * * *
$$

Shaikh A, Cevallos ML, Lan F, Daniel JP. Multiple nocardial brain abscesses in an immunocompromised patient with myasthenia gravis. International Journal of Case Reports and Images 2013;4(1):11-14.

$$
* * * * * * * * *
$$

doi:10.5348/ijcri-2013-01-249-CR-3

\section{INTRODUCTION}

Nocardia can be found almost universally in soil and plants. Nocardia was first identified by Edmund Nocard in 1888 in bovine farcy. The first human disease was described by Eppinger in 1890. Nocardiosis is an opportunistic infection caused by gram positive, weakly acid-fast, filamentous, aerobic organisms $\left[\begin{array}{ll}1, & 2\end{array}\right]$. Nocardia asteroides is the most common species to cause infection in humans [3-5]. There are at least thirteen, Nocardia spp. but N. asteroides, N. farcinia and N. nova (N. asteroides complex) constitute about $80-90 \%$ of the total cases $[3,4]$. Though nocardiosis is a relatively rare bacterial infection, it is frequently associated with immunosuppression. The majority of infections occur in patients with weakened cellmediated immunity. Infected population generally comprises those who have received bone marrow or 
solid organ transplantation, patients on immunosuppressive therapy, those with human immunodeficiency virus/acquired immunodeficiency syndrome (HIV/AIDS), patients on long-term steroid therapy and those with malignancies $[3,6]$.

\section{CASE REPORT}

A 32-year-old female was brought to the emergnency room (ER) with complaints of severe headache, nausea, vomiting and altered mental status. A diagnosis of myasthenia gravis had been made three years earlier, associated mainly with diplopia. Her medications included $60 \mathrm{mg}$ of pyridostigmine and $60 \mathrm{mg}$ of prednisone daily. No history of seizures, fever or photophobia was obtained. On neurological examination, she was confused and incoherent. There was generalized weakness with no focal neurological deficits and preserved deep tendon reflexes. The patient's mental status deteriorated to the point that she had to be intubated and placed on mechanical ventilation. Noncontrast computed tomography (CT) scan of brain showed multiple ring lesions in both the hemispheres. On review of the chart, it was found that the patient had been recently discharged from surgical service after drainage of left shoulder abscess. Wound culture at that time had grown diptheroid organisms, and the patient was discharged home on oral augmentin after a brief course of intravenous vancomycin in the hospital.

In view of the immunocompromised status and recent history of shoulder abscess, metastatic cerebral abscess was ascertained to be the most likely cause, and the patient was started on empiric antimicrobial therapy including coverage for possible toxoplasmosis (ampicillin, vancomycin, ceftriaxone, metronidazole and trimethoprim-sulfamethoxazole (TMPSMX)).

Magnetic resonance imaging (MRI) of the brain with contrast was obtained for better evaluation of the cerebral lesions and showed bilateral occipital ring enhancing lesions (Figure 1, 2). Initial set of blood cultures remained negative after $48 \mathrm{hr}$. Toxoplasma titers were negative. A stereotactic biopsy of one of the cerebral lesion was done and frank pus was aspirated, however, gram stain failed to reveal any organisms. While awaiting biopsy culture results, empiric antibiotic coverage was continued with the exception of ampicillin.

Serial interval neurological examinations continued to be nonfocal and unchanged from presentation (Figure 3). Computed tomography scann of the chest and abdomen showed no abnormalities. The patient's mental status improved gradually and the patient was successfully weaned off the ventilator on the seventh day of hospitalization. Aerobic and anaerobic bacterial cultures of the brain biopsy aspirate continued to show no growth. However, on the 19th day of hospitalization, fungal cultures were reported to be positive for Nocardia. At this time, staphylococcal and anaerobic coverage was discontinued and patient was continued on IV ceftriaxone and IV TMPSMX.
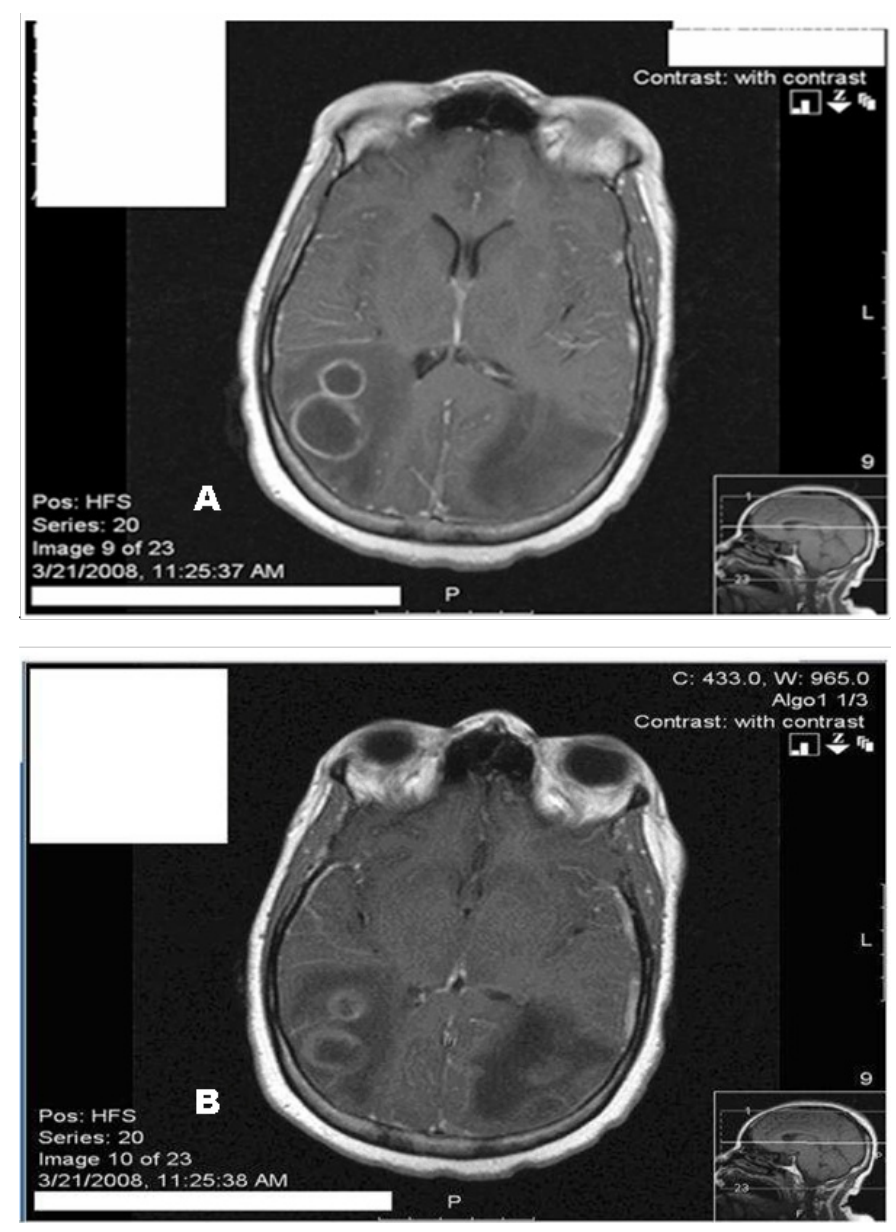

Figure 1: (A, B) Magnetic resonance imaging of the brain with contrast (gadolinium) at presentation, showing bilateral occipital ring enhancing lesions.

Speciation and further identification confirmed the organism to be Nocardia asteroides sensitive to the antibiotic regimen.

A permanent intravenous access was established as prolonged antibiotic therapy was necessary and the patient was discharged home with plan to continue intravenous antibiotics for total of six months according to current guidelines.

The patient was subsequently followed-up in the out patient clinic and is doing well with some complaints of occasional sharp pain in left parietal area and no focal neurological deficits. The patient is presently in the fifth week of antibiotic therapy.

\section{DISCUSSION}

Nocardiae are gram positive, aerobic actinomycetes found naturally in the soil, air and sewage. Nocardia asteroides is the predominant species and the one most commonly associated with disseminated disease. Although there have been many reports of disseminated nocardiosis in immunocompromised patients, primary cerebral nocardiosis is a very rare presentation. 

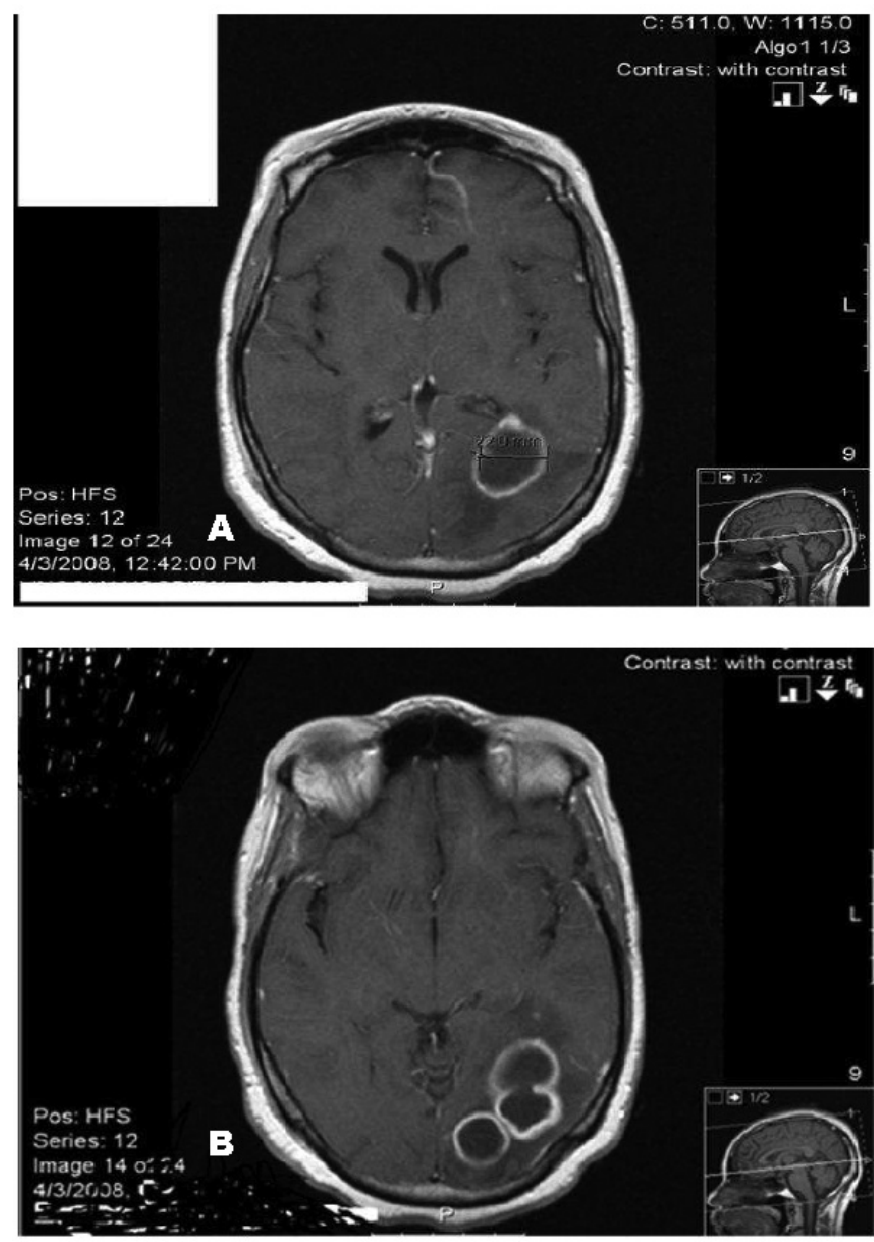

Figure 2: (A, B) Magnetic resonance imaging of the brain with contrast (gadolinium) shows punctate pontine lesions slightly larger than on the prior study. Bilateral occipital abscesses have slightly decreased in size and edema as compared to the prior MRI.

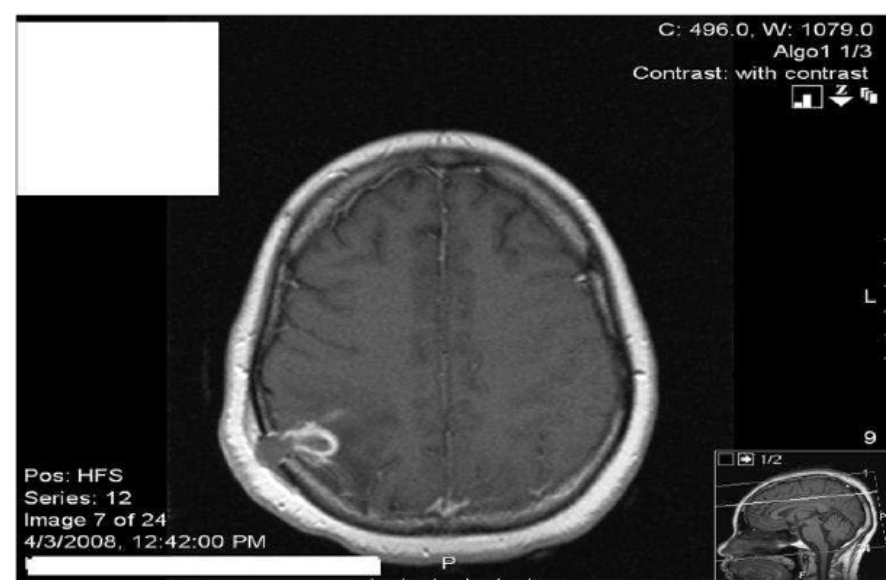

Figure 3: The magnetic resonance imaging of the patient post right occipital craniotomy with biopsy of one of the lesions and aspiration of pus.

Nocardial abscess associated mortality is reported to be three times higher than in patients with other bacterial brain abscesses. Management of nocardial brain abscess remains a clinical challenge and is associated with very high morbidity and mortality rates (about 90\%) [7-9]. A definitive diagnosis can only be made with the isolation and identification of the organism by invasive procedures and therefore, a high index of suspicion is required as early institution of therapy can be lifesaving. Cultures can take up to 13 weeks to grow and speciation is difficult.

All treatment modalities for nocardiosis generally involve TMPSMX. However, there have been isolated reports of benefit from amikacin and ceftriaxone. Aspiration has been recommended as the preferred modality initially for nocardial brain abscess, with aggressive surgical management being reserved for the small proportion of patients who do not respond to minimally invasive surgery.

Our patient was a housewife with no history of recent travel, trauma or engagement in water sports. Infection with Nocardia was therefore most likely via the respiratory tract, which is the generally accepted mode of inoculation. Her recent shoulder abscess was in all probability a Nocardial infection as Nocardia can be misidentified for diptheroid organisms owing to analogous gram staining and morphological features. This fact, coupled with the immunocompromised status raised the suspicion for nocardial abscess initially. Nocardial cerebral abscess can give rise to focal neurological deficits depending on the intracranial location. In our patient, no motor or sensory focal deficits were noted on serial neurological examinations as all of the abscesses were localized to the occipital and posterior cortex. A good clinical response to the combination therapy with intravenous ceftriaxone and TMPSMX was noted.

\section{CONCLUSION}

We report a single case of multiple nocardial brain abscesses in an immunocompromised patient on long term corticosteroid therapy for myasthenia gravis. Nocardiosis frequently goes undiagnosed initially as the patient would have either responded to empiric antimicrobial treatment given for some other reason or because Nocardia can very easily be mistaken for nonpathogenic microorganisms (diphtheroids) and discarded on account of its morphological similarities. The diagnosis requires a high clinical suspicion with early tissue and microbiological diagnosis. Prolonged antimicrobial therapy of 6-12 months and serial imaging is key in treatment and prevention of relapse.

$$
* * * * * * * * *
$$

\section{Acknowledgements}

We would like to acknowledge and extend our heartfelt gratitude to the following persons who have made the completion of this case report possible: Rajan Khanna, MD; Cesar A Lopez, MD

\section{Author Contributions}

Ayesha Shaikh - Conception and design, Acquisition of data, Analysis and interpretation of data, Drafting the 
article, Critical revision of the article, Final approval of the version to be published

Maria Lola Cevallos - Conception and design, Acquisition of data, Analysis and interpretation of data, Drafting the article, Critical revision of the article, Final approval of the version to be published

Fang Lan - Acquisition of data, Analysis and interpretation of data, Drafting the article, Final approval of the version to be published

Jean Pratt Daniel - Conception and design, Analysis and interpretation of data, Critical revision of the article, Final approval of the version to be published

\section{Guarantor}

The corresponding author is the guarantor of submission.

\section{Conflict of Interest}

Authors declare no conflict of interest.

\section{Copyright}

(C) Ayesha Shaikh et al. 2013; This article is distributed under the terms of Creative Commons Attribution 3.0 License which permits unrestricted use, distribution and reproduction in any means provided the original authors and original publisher are properly credited. (Please see www.ijcasereportsandimages.com /copyright-policy.php for more information.)

\section{REFERENCES}

1. Cortese I, Nath A. Case 11: a young woman with ringenhancing brain lesions. MedGenMed 2006 Jan $5 ; 8(1): 3$.
2. Mamelak AN, Obana WG, Flaherty JF, Rosenblum ML. Nocardial brain abscess: treatment strategies and factors influencing outcome. Neurosurgery. 1994;35(4):622-31.

3. Sereti I, Holland SM. Disseminated nocardiosis in a patient with X-linked chronic granulomatous disease and human immunodeficiency virus infection. Clin Infect Dis 2001;33(2):235-9.

4. Barnaud G, Deschamps C, Manceron V, et al. Brain Abscess Caused by Nocardia cyriacigeorgica in a Patient with Human Immunodeficiency Virus Infection. J Clin Microbiol 2005;43(9):4895-7.

5. Wakhlu A, Agarwal V, Dabadghao S, Prasad KN, Nityanand S. Nocardiosis in patients of chronic idiopathic thrombocytopenic purpura on steroids. J Assoc Phys India 2004;52:591-3.

6. Talwar P, Chakrabarti A, Ayyagari A, et al. Brain abscess due to Nocardia. Mycopathologia 1989;108(1):21-3.

7. Skiest DJ. Focal Neurological Disease in Patients with Acquired Immunodeficiency Syndrome. Clinical Infectious Diseases 2002;34(1):103-15.

8. Lee GY, Daniel RT, Brophy BP, Reilly PL. Surgical treatment of nocardial brain abscesses. Neurosurgery 2002;51(3):668-71.

9. Elmaci I, Senday D, Silav G, et al. Nocardial Cerebral Abscess Associated with Mycetoma, Pneumonia, and Membranoproliferative Glomerulonephritis. J Clin microbiol 2007 June;45(6):2072-4.
Access full text article on other devices

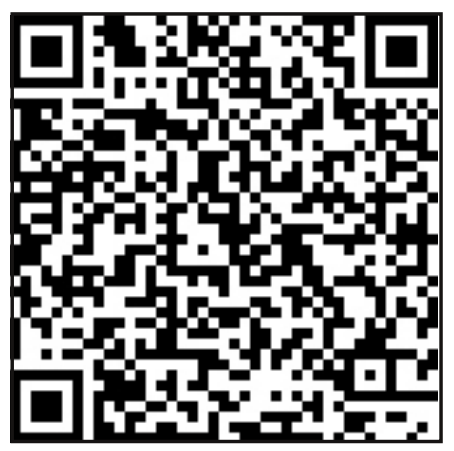

Access PDF of article on other devices

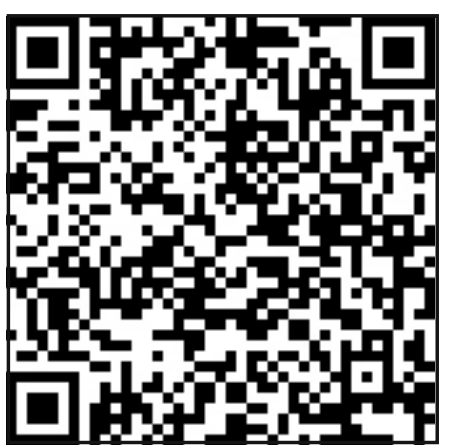

